\title{
Neuro-Fuzzy based Call Admission Control for next Generation Mobile Multimedia Networks
}

\author{
Sanjeev Kumar, Krishan Kumar
}

\begin{abstract}
As the demand of the mobile users are increasing day by day, wireless/mobile multimedia networks still need advancement in terms of, reliable traffic performance, link availability, efficient bandwidth utilization, and user mobility, that can attain extremely consistent wireless communication and data transmission over the networks. Due to the emerging demand of multimedia services a high-speed network and call admission control (CAC) scheme is required, which not only guarantees the quality of services (QoS) for new and handoff calls but also results in optimum resource utilization in bursty traffic network environments. The main objective of this integrated neural fuzzy based CAC scheme is to improve QoS with decent resource allocation, such that it minimizes the probability of call dropping and call blocking in mobile multimedia networks. The proposed neural fuzzy CAC scheme is a hybrid approach that integrates the semantic rule ability of fuzzy logic (FL) controller and self-training capability of a neural network $(N N)$ which is further enhanced to construct an efficient computational model for traffic control and fair radio resources allocation for new calls and handoff calls. The simulation results conclude that a neural fuzzy based CAC can achieve minimal call dropping probabilities and maximum resource utilization in high-speed networks as compared to fuzzy logic based CAC and conventional CAC or existing CAC schemes.
\end{abstract}

Keywords: Mobile Multimedia Networks, Call Admission Control, Fuzzy logic, Neural Networks, self-organization map, Quality of Service, Call dropping probability.

\section{INTRODUCTION}

Today, with the growing demand for mobile users advanced multimedia services, there is a need for high-speed mobile multimedia networks to support various multimedia applications and services. High-speed networks use mobile/wireless communication networks to offer wireless communication along with static and dynamic hosts, which is capable to handle bursty traffic and maintaining various quality-of-service (QoS) like latency, packet loss, load, signal strength, user mobility, and channels requirements, etc. In a multimedia high-speed network environment, efficient channel assignment and CAC schemes are necessary to obtain a better resource management scheme with an appropriate QoS level for end users.

Revised Manuscript Received on October 30, 2019.

* Correspondence Author

Sanjeev Kumar*, Computer Science, Gurukula Kangri Vishwavidyalaya, Haridwar, India. Email: sanjeevnmc83@gmail.com

Krishan Kumar, Computer Science, Gurukula Kangri Vishwavidyalaya, Haridwar, India. Email: krishankumar1614@gmail.com

(C) The Authors. Published by Blue Eyes Intelligence Engineering and Sciences Publication (BEIESP). This is an open access article under the CC BY-NC-ND license (http://creativecommons.org/licenses/by-nc-nd/4.0/)
One of the best technique that combines the multimedia services for high-speed mobile multimedia networks, is Asynchronous transfer mode (ATM) [1].

A fast speed network like $4 \mathrm{G}$ and $5 \mathrm{G}$ networks are considered to be the fastest network technology that is capable to provide a high-speed data transfer rate up to 450 Mbps and efficient quality of services for real-time application in multimedia networks [2]. The increasing population of advanced mobile multimedia device, the user always seek the best connection anywhere anytime in order to achieve efficient policy to maintain seamless handoff between heterogeneous networks and QoS requirement.

Consequently, it is too much hard to select the best networks to receive a new call without interrupting the QoS level of ongoing calls. Due to the rapidly increasing number of the mobile access point, continuous changes in channels propagations, sudden changes in network load and random mobility of the user, a high-speed network (4G \& 5G) suffers from uncertainty and imprecision that is subject to decision error, which degrades the system performance [3-7]. Therefore there is a need to develop a framework that dynamically monitors the traffic flows according to a sudden change in network load conditions and ensure fair admission control and efficient service delivery of all types of call requests.

CAC is one of the essential channel allocation and decision-making technique which ensures desired QoS by limiting the use of network resources [8]. However, the decision making for CAC is a high demanding research topic due to the finite number of radio resources, random user mobility, and uncertain multimedia network traffic conditions. To provide a number of integrating services with certain QoS parameters like network lose rate, signal quality, jitter, call blocking and call dropping, a CAC scheme is needed which restrict the number of new connections to minimize the traffic jam and handoff dropping probability. The admission controller decides either to admit or deny new call requests based on the present traffic load and unoccupied radio resources to continue the QoS for a new call or handoff calls without violating the QoS of ongoing calls. It is because of mobile users are more conscious of call dropping instead of to new call blocking [3] [9]. An efficient CAC policy is required to provide a guarantee that satisfies all the QoS requirements with respect to different traffic conditions [10]. So far several improved models and assumptions have been broadly used to manage the issues of CAC and QoS in a high-speed multimedia network using Markov models, queuing models and expert systems, etc.

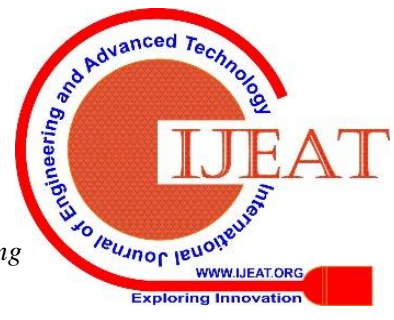


in the last decades. This paper offers a combined approach of neuro-fuzzy based CAC (NFCAC) model, which join the parallel computing and learning capabilities of neural network with semantic control power of fuzzy logic (FL) to make the CAC decision in order to maintain the QoS provision. The beauty of the neuro-fuzzy model is its adaptable intelligent system, training from experience and human comprehension capability and strict mathematical modeling requirements that can be simplified by fuzzy IF-THEN type rules, which enhance the wireless communication and data transmission speed over the high-speed networks like 4G and 5G.

The NFCAC model uses the self-training function of the neural network (NN) to minimize CAC's general policy decision-making errors, generated from unpredictable variations in modeling, approximation, and system traffic. The implementation of an FL controller rule structure is used to avoid error handling because of erroneous training and to shorten training time. A neuro-fuzzy network is considered as a well-organized network. The suitable input parameters have been selected and prepared a regular fuzzy rule blueprint for the NFCAC method so that it not only offers a solid structure to imitate the knowledge of experts embedded in previous traffic control algorithms but also a smart mathematical algorithm has been developed as well for traffic control.

A lot of research work has been done that deals with CAC related problem in wireless networks by using FL and NN respectively. Neural-fuzzy model has been broadly used to improve the performance of existing CAC policy in high-speed mobile networks. FL has the capability to deals with the problem of real-world imprecision, vague, ambiguous, uncertain data and burst environment by using mathematical framework [11-15]. The comparative analysis of the simulation result shows that the proposed fuzzy approach greatly improves system performance as compared to existing typical CAC schemes. However, there is currently no clear and universal technology that maps existing traffic control knowledge to fuzzy logic controller design parameters. So the self-learning capabilities of NN ought to be included into FL controllers to understand the design process to achieve more desirable control result. This paper uses a combined approach of FL and NN to deal with the problem of traffic mobility, system resource utilization, and QoS by using CAC scheme.

\section{PREVIOUS WORK}

In the past few years, many studies have used fuzzy logic (FL) and neural networks (NN) to manage CAC-related issues in mobile multimedia networks. The neuro-fuzzy model has been widely used to improve the performance of existing CAC policy in high-speed mobile multimedia networks. The previous study of the neural fuzzy controller has been shown that it has the capacity to handle the complex problems of the system. In this regard, FL has the capability to deal the problems of real-world imprecision, whereas the self-learning ability of self-organization map (SOM), a famous NN model is used to specify the association into input network traffic and system capabilities. The NN makes FL more powerful by providing an accurate data set for training.

This permits the fuzzy system to be optimized to exactly match the training data set [16]. When a neural-fuzzy based strategy used in mobile multimedia networks, each cell is treated as a separate controller, representing a unique behavioral model based on user mobility and cell geographic attributes. Therefore, even if the available bandwidth requirements vary according to changes in call load status, but the correct QoS parameters must be maintained to reduce the probability of new call blocking and handover loss [1], [3], [11-16]. Fuzzy logic systems have been commonly used to address CAC associated issues in mobile multimedia networks that have been extensively studied in the literature review.

The fuzzy set theory provides a strong computational model to handle real-world inaccuracies. Fuzzy methods show flexible performance and can adapt to dynamic, incorrect and realistic environments. [1], [11], [16]. Ravi sandal et. al. [13] developed a neuro-fuzzy based CAC for constant and non-continuous traffic in CDMA cellular network to handle the handoff prioritization and channel allocation for handoff calls (both type) and shows the comparison between soft handoff and hard handoff.

In [17], the authors proposed a fuzzy-neural-based CAC method combining fuzzy logic control function and learning function of recurrent neural redial basis function and developed a smart system capable of processing incoming traffic in the diverse mobile network environment.

In [18-19], has been proposed a fuzzy-based CAC method to maintain the QoS level of ongoing calls and estimate the system performance using the shadow cluster concept (SCC). An integrated fuzzy logic based CAC for 4G networks in order to improve the QoS provision with the help of Karnik-Mendel (KM) and Wu-Mendel (WM) model and also compare type-1 fuzzy logic with type-2 fuzzy logic controller [20]. The fuzzy logic system has been a great deal of attention during last two decades and has achieved great success in many different real-world application like ATM networks, wireless mobile network, wireless sensor networks, and traffic congestion control. The literature survey has shown that previously the researcher has faced so many difficulties to handle the uncertainties because of a hard decision boundary, but with the advancement of FL, logic systems are characterized by membership functions with soft decision boundaries [21-24].

In [25], the author proposed mobility based CAC using an artificial neural network, in this the mobility of user is found with the previous and present user mobility history by using error back propagation model and base on the user mobility the allocation of the resource are made by queuing model. Here the neural network model trained to estimate the required bandwidth to accept voice and data calls in different QoS requirements.

In [26], a CAC scheme based on dynamic channel assignment has been proposed. Error Backpropagation and Hopfield neural networks, two well-known NN models were used to improve the QoS and minimized call dropping probability and call blocking probability in mobile multimedia networks. 
In the first phase, the error back propagation model is used to perform the effects of location prediction and traffic mobility performance estimation. In the second stage, the Hopfield neural network based dynamic channel allocation (DCA) scheme has been employed to increase the performance of the existing dynamic channel allocation scheme. In [27], the author's group investigate the new flexible use of the Hopfield neural network to perform dynamic call admission control. The self-learning approach of the neural network has been used to design a CAC in [28], [29]. According to the literature survey most of the proposed CAC approach, select the number of users as input parameter according to their types of service. As the network traffic or complexity of the system increases, the dimension and the training time of NN also increases. Therefore, the applications of NN with respect to CAC are not much more capable to handle complex traffic environment, large traffic congestion, and complex network traffic source, etc. However neural-fuzzy logic based CAC policies have more capacity to handle CAC activities and maintaining QoS provisioning for the mobile users.

The objective of this research paper is to develop a neuro-fuzzy-based CAC model that implements appropriate channel allocation scheme that covers the maximum geographical area in order to enhance QoS from the viewpoint of continuous service availability in high-speed Mobile Multimedia Network. The simulation result shows that the NFCAC model offers efficient system resource utilization, high learning speed, user mobility, quality of services and minimize the call blocking probability (CBP) and call dropping probabilities (CDP). In order to verify the effectiveness of the proposed scheme, the simulation results are compared with existing neural-fuzzy based CAC methods.

\section{NEURO-FUZZY CALL ADMISSION CONTROLLER (NFCAC)}

Fuzzy Logic (FL) was firstly introduced by Lotfi A. Zadeh, which represent uncertain and imprecise knowledge. FL is used to handle the complex problems and represent there result in the form of range rather than true and false. A fuzzy set is an amalgamation of elements and each component of this set is specified by a fuzzy membership function with membership range from 0 to 1 [30]. The idea of the FL has been mainly used to characterize the performance of nonlinear systems. Fuzzy based rules effectively handle and represented the nonlinear system. [16], [31]. FL is a rule-based architecture associated with four main modules: fuzzification, inference engine, rule base, and defuzzification. In this case, a fuzzy set $\mathrm{F}$ has been defined in the discourse world $\mathrm{U}$ by defining the language variable $x$ in $\mathrm{U}$

$T(x)=\left\{T_{x}^{1}, T_{x}^{2}, T_{x}^{3} \ldots \ldots \ldots T_{x}^{k}\right\} \quad$ and $\quad M(x)=$ $\left\{M_{x}^{1}, M_{x}^{2}, M_{x}^{3} \ldots \ldots \ldots M_{x}^{k}\right\}$, where $T(x)$, the fuzzy input is set of $x$ and $M(x)$, a set of rules that are connected with each input with their meaning. According to fig. 1, the fuzzifier convert an input $x_{i}$ of fuzzy set $T_{x}^{k}$ with a degree of membership function $M_{x_{i}}^{k_{i}} i=1 \ldots \ldots \ldots \ldots . .$. The fuzzy rule base stores control information of domain that specify fuzzy IF-THEN rule for a set of linguistic statements, which define a FL based association between m-dimensional inputs $x_{i}$ and n-dimensional outputs $y_{i}$. As the fuzzification process completed, the inference engine generates a decision parameter for deriving the input language variable values of $T\left(x_{i}\right)$ from the fuzzification to obtain the output linguistic variable of $\mathrm{T}\left(\mathrm{y}_{\mathrm{i}}\right)$, namely fuzzy IF-THEN rules. When the output linguistic variable of $\mathrm{T}\left(\mathrm{y}_{\mathrm{i}}\right)$ is available to the defuzzifier, then defuzzification function generates a non-fuzzy values form the output $y_{i}$ that constitutes decision. The fuzzy logic controller contains domain information from previously developed schemes [31, 32].

In 1943, Mc Culloch Pitts introduced an artificial neural network (ANN) that are organized in layers. The layers or nodes of the neural networks are interconnected to each other, which contains an activation function

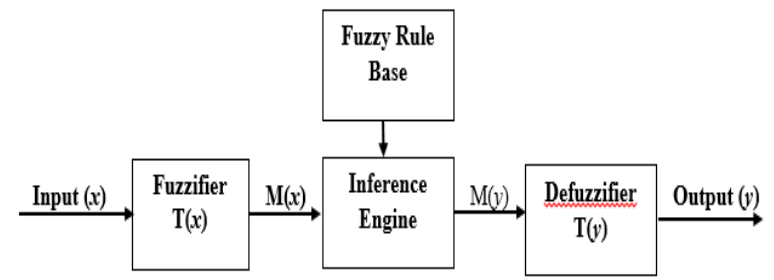

Fig. 1. Structure of Fuzzy logic Control System.

Here, a multilayer feedforward neural network has been used that includes various input layers, one or more hidden layers, and output layers. In this network, information is only moved between different non-linear processing elements called nodes that go from the input node to the hidden node and then to the output node in the forward direction. Each node of the ANN layer consists of multiple neurons that are fully connected to their neighboring layer neurons and have continuously changing weights. According to Fig. 2, the output of one node is connected to the input of another node with its associated weight. Each node calculates the sum of the input weights and produces output through a predefined activation function [25].

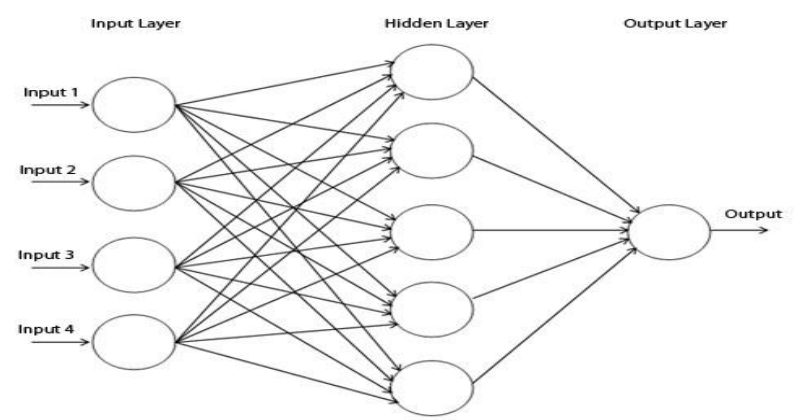

Fig.2. Architecture of multilayer feedforward neural network

In addition to this a feed-forward neural network NN $\left(V_{i}, W_{i}\right)$, has been defined, where $V_{i}$ represents the set of the input vector and $W_{i}$ represents the set of weight vectors. The values of both vectors will be modified by learning rules. When feed forward $\mathrm{NN}\left(V_{i}, W_{i}\right)$ is train, it produce an approximate output that is close to the actual output. The neural network can't contains accurate information of the system, due to unstructured network architecture [1].

This proposed NFCAC model is a combination of two soft computing techniques NN and FL. Here the self-trained and computational capabilities of NN brings connected into FL system to obtain the excessive level human reasoning and thinking of fuzzy logic system for NN.

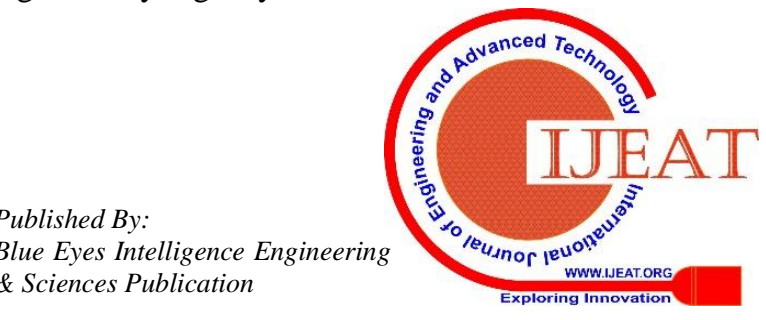


Basically, the integration system of neural fuzzy use a multilayer network to understand the FL system behavior [13-15] [33]. Now it is a well-organized network that can contains accurate system information for traditional schemes.

\section{A. Design of NFCAC}

In this, we used five layered neural fuzzy network architecture to design a neuro-fuzzy call Admission controller. As represented in fig. 3, the NFCAC controller is a five-layered architecture, where layer 1 nodes received all the input linguistic variables and node 5 produces two linguistic outputs. One output is supplying the tanning data to the network, to produce desired output and the second is used for transporting the decision signal to produce the actual output for the network. Furthermore, layer 2 and layer 4 nodes are considered as terms nodes that act as a fuzzy membership function for each linguistic variables. Every node of layer 3 define a fuzzy rule and when all these nodes combine they design a fuzzy inference rule base. The working of the inference engine is served by layer 3 and layer 4 connections, layer 3 connections show the fuzzy rules nodes and layer 4 connections shows the results of the rule nodes. The links of layer 2 and layer 5, represents the complete connection between the input linguistic nodes and its parallel terms nodes.

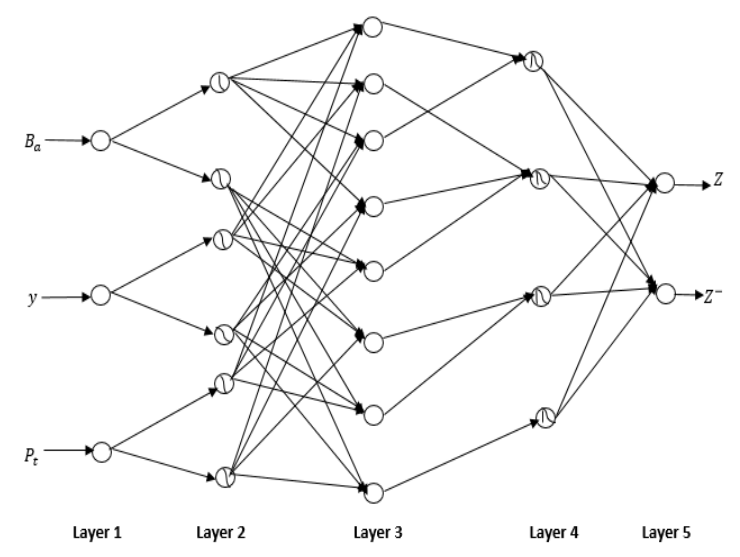

Fig. 3. The architecture of the NFCAC controller.

According to fig. 3, the NFCAC controller having three inputs as available bandwidth $B_{a}$, a network traffic jam signal $\mathrm{y}$, cell loss ratio $P_{t}$ and an output decision symbol $\mathrm{Z}$, which shows the acceptance and rejection of the new call request. The available bandwidth can be represented by the equation (1), and widely used in the fairly bandwidth distribution based CAC policies.

$B_{a}=B_{t}($ Total Bandwidth $)-B_{u}($ Used Bandwidth) (1)

The network traffic jam indicator $y$ shows the level of traffic jam currently available in the network and provides accurate knowledge of the network system. The cell loss ratio $P_{t}$ is represents the feedback based system performance, which has the capability of modifying itself to provides secure and powerful operation.

Each layer of NFCAC system is associated with the fuzzy inference process. The NFCAC controller has a network input function $f n_{i}^{(k)}\left(u_{i j}^{(k)}\right)$ and an output activation function $a_{i}^{(k)} f n_{i}^{(k)}$ for all nodes $i$ to $\mathrm{k}$, where $u_{i j}^{(k)}$ represent a possible set of input to node $i$ in layer $k$ form node $j$ in layer $(k-1)$. The details of the five layers are given below [1], [4], [13-14].
Layer 1. (Input Layer): In this layer 1 neurons communicates with the crisp signals of the next layer. For fuzzy linguistic input parameters, $B_{a}, y$ and $P_{t}$ is the generalized representation of $T_{x}^{k}$, where $x$ linguistic input parameter and $k$ is the current layer. In NFCAC $B_{a}, y$ and $P_{t}$ are defined as follow

$f n_{i}^{(1)}\left(u_{i j}^{(1)}\right)=u_{i j}^{(1)} \quad$ and $a_{i}^{(1)}=f n_{i}^{(1)}$

Where $u_{11}^{(1)}=B_{a}, u_{22}^{(1)}=\mathrm{y}, u_{33}^{(1)}=P_{t}$, and $1 \leq i \leq 3$ and $1 \leq$ $k \leq 5$.

Layer 2. (Fuzzification Layer): In layer 2 nodes, fuzzifier is used to receive crisp inputs and defining fuzzy rules. The term used to define the available bandwidth with a set of $T\left(B_{a}\right)=\{$ Low $(\mathrm{L})$, Medium $(\mathrm{M})$, High $(\mathrm{H})\}$, the term set for network traffic load variables is defined as $\mathrm{T}(y)=\{\operatorname{High}(\mathrm{H})$, Medium (M), Low (L)\}, which shows the network congestion state of the system, the system can be in any one of the congestion state represented by high, medium and low, high represent the congestion full networks whereas low congestion free networks. The term set for the cell loss ratio is define as $\mathrm{T}\left(P_{t}\right)=\{\operatorname{High}(\mathrm{H}), \operatorname{Medium}(\mathrm{M})$, Low $(\mathrm{H})\}$, which is one of the most important QoS requirement parameter. For all these above terms set we have six node in this layer. In layer 2 each node performed a bell-shaped activation function of a membership neurons, which specifies the neuron's fuzzy set. The activation function is defined as

$$
\begin{gathered}
f n_{i}^{(2)}\left(u_{i j}^{(2)}\right)=-\frac{\left(u_{i j}^{(2)}-m_{j n}^{(I)}\right)^{2}}{\sigma_{j n}^{(I)^{2}}} \\
a_{i}^{(2)}=e^{f n_{i}^{(2)}}
\end{gathered}
$$

Where $u_{i j}^{(2)}=a_{j}^{(1)}, 1 \leq i \leq 6, j=\left\lceil\frac{i+1}{2}\right\rceil$ and $m_{j n}^{(I)}$ and $\sigma_{j n}^{(I)}$ are define the mean (or center) and standard deviation (or width) for every nth term of the crisp input set from node $\mathrm{j}$ in input layer. If the $i^{t h}$ node is odd then set $n=1$, otherwise set $n=2$.

Layer 3. (Rule base Layer): In this layer, every neuron coincides with a unique fuzzy rule. The links between fuzzy rules neurons get input from the fuzzification neurons that show the necessary matching conditions of fuzzy control rule. The fuzzy set theory says that the fuzzy rule base can be represented by a fuzzy set with dimensions $T\left(B_{a}\right) \times \mathrm{T}(y) \times \mathrm{T}$ $\left(P_{t}\right) \times T(x)$ which denotes the number of inputs $\operatorname{in} T(x)$.

In this layer, there are eight rule nodes. In a neuro-fuzzy system, each rule node can be executed by the fuzzy AND logic operation defined as follow

$f n_{i}^{(3)}\left(u_{i j}{ }^{(3)}\right)=\min \left(u_{i j}{ }^{(3)} ; \forall j \in P_{i}\right)$

$$
a_{i}^{(3)}=f n_{i}^{(3)}
$$

Where $u_{i j}{ }^{(3)}=a_{j}{ }^{(2)}$ and $P_{i}=\left\{\mathrm{j} \mid\right.$ all $\mathrm{j}^{\text {th }}$ preconditioned nodes of the $\mathrm{i}^{\text {th }}$ rule $\}, 1 \leq i \leq 8$.

Layer 4. (Output membership Layer): In layer 4, the neurons are represented by the fuzzy sets and used as the resultant of fuzzy based rules. All inputs of the output membership neurons are combined with the help of fuzzy union operations. This operation can be realized by probabilistic OR operations. In this layer, down-up and up-down are two operating modes.

\section{Published By:}


The down-up is utilized to decide the result matching of fuzzy inference rules pertinent to the input. Based on the output set (up-down) of linguistic variables, a soft admission decision set can be defined as $T\left(z^{-}\right)=\{$“"Reject" (R), "Weak Reject" (WR), "Weak Accept" (WA) "Accept"(A)\}.Therefore, NFCAC controller may have describe the acceptance/rejection decision boundary of Call. This layer consist of four nodes. To integrates the firing strength of fuzzy rules, each node implement a fuzzy OR operation. Thus, we define

$$
\begin{aligned}
& f n_{i}{ }^{(4)}\left(u_{i j}{ }^{(4)}\right)=\max \left(u_{i j}{ }^{(4)} ; ; \forall j \in C_{i}\right. \\
& a_{i}{ }^{(4)}=f n_{i}{ }^{(4)}
\end{aligned}
$$

Where $u_{i j}{ }^{(4)}=a_{j}{ }^{(3)}$ and $C_{i}=\{\mathrm{j} \mid$ all $\mathrm{j}$ that contains the same resultant of the ith node in the input term set of $\left.z^{-}\right\}$, $1 \leq i \leq 4$.

The up-down is applied during the training phase of the system by providing the output data set to them. The nodes in the present layer and the links in layer 5 have some common function like as in layer 2. Each node is represented by a bell-shaped fuzzy function defined as

$$
\begin{array}{r}
f n_{i}^{(4)}\left(u_{i j}^{(4)}\right)=-\frac{\left(u_{i j}^{(4)}-m_{j}^{(0)}\right)^{2}}{\sigma_{j}^{(0)^{2}}} \\
a_{i}^{(4)}=e^{f n_{i}^{(4)}}
\end{array}
$$

Where $u_{i j}^{(4)}=a_{j}^{(5)}$, received by the up-down operating mode in layer 5 , and $m_{j}^{(O)}$ and $\sigma_{j}^{(O)}$ are ddefine the mean (or center) and standard deviation (or width) for every jth term of $z^{-}$, respectively, $1 \leq i \leq 4, j=1$.

Layer 5. (Defuzzification Layer): This layer is the output layer where every neuron shows the one output of the neuro-fuzzy system.

Here the output fuzzy set is taken from two nodes, the first node performs a down-up operation and second performs up-down operation respectively. The first node performs down-up operations for the real decision parameter $z^{-}$for the acceptance/rejection of the call. Each node and its lines works like as defuzzifier. The defuzzification process is calculated by the following function

$$
\begin{gathered}
f n_{i}^{(5)}\left(u_{i j}{ }^{(5)}\right)=\sum_{j=1}^{4} m_{j}^{(0)} \sigma_{j}^{(O)} u_{i j}{ }^{(5)} \\
a_{i}^{(5)}=U\left(\frac{f n_{i}^{(5)}}{\sum_{j=1}^{4} \sigma_{j}^{(O)} u_{i j}^{(5)}}-z_{a}\right)
\end{gathered}
$$

Where $u_{i j}{ }^{(5)}=a_{j}{ }^{(4)}, i=1, z_{a}$ is a decision threshold and

$$
U(x)=\left\{\begin{array}{lr}
1, & \text { if } x \geq 0 \\
0, & \text { otherwise. }
\end{array}\right.
$$

The above function shows that the new call will be accepted if $z^{-}=a_{1}{ }^{\left({ }^{5)}\right.}$ and $z^{-}=1$. The second node performs the up-down at the tranning time of the system.It supplies the desired output signal into the NFCAC controller to updates the weigths optimally. For this type of node we defined

$f n_{i}^{(5)}\left(u_{i j}{ }^{(5)}\right)=f n_{i}^{(5)}\left(u_{i i}{ }^{(5)}\right)$ and $a_{i}^{(5)}=f n_{i}^{(5)}$

\section{A. Self-organizing map and hybrid learning algorithm}

Kohonen's self-organizing map (SOM) is a unique and most renewed artificial neural network (ANN) method for dimension reduction. It can be either supervised or unsupervised. It can be either supervised or unsupervised. As the name implies the Self-Organizing Maps is a combination of "self-Organizing" and "Maps". "Self-Organizing" is used to learn by itself in unsupervised modest learning and "maps" is used to successfully mapping the weights to given input data. The input patterns in the SOM are represented by nodes in order to learn the input patterns. SOM is also known as "Featured Maps". Here the main objective of the SOM is to transfer an incoming linguistic input pattern of $\mathrm{m}$-dimensional values to a fewer dimensional output mapped values, in order to maintain the original topological relations them. The output neurons of the network fight themselves to be activated in order to obtain resultant neuron. Only a single neuron per group is selected at a time. From the output neuron only one neuron is selected (that wins the fight or competition) is known as a winner neuron or a winner-takes-all neuron. In [37] the author describes the use of self - organization map (SOM) in 4G mobile network to make the mobile network system more flexible. In order to examine and manipulate the working model of SOM, the author used entropy- based complexity matrix to replace the centralized resource assignment with highly decentralized controlled resource assignment in the $4 \mathrm{G}$ mobile network [38]. One of the authors in [39] used Kohonen's self-organizing map to provide an efficient channel assignment during the mobile network operations in a very effective manner. This approach is partitioned into two phases, in the first phase the accurate location of the user finds out by the Manhattan-like network and in the second phase allocations of the resources are made by SOM. This improves the quality of services and performance of the mobile network by applying some restrictions during the channel assignment for the new calls and handoff calls.

In this paper for the design of NFCAC controller, we associate FL and self-organizing map to develop a hybrid learning algorithm. In this algorithm, a two-stage learning method is implemented. The first phase employs the use of self-organized learning scheme for the construction of rules and to identify the starting membership functions. Stage two adopts supervised learning method for the ideal adjustment of the membership functions to achieve desired outputs. In addition to this, the size of each input/output variable set, fuzzy control rules, and training data must be defined and available for the learning process.

The process for construction of the training data set is depicted below;

In order to prepare the training data set for new calls the NFCAC requires a fuzzy bandwidth calculator that calculates the required bandwidth $B_{e}$, the network resource calculator that calculate the total available bandwidth $B_{a}$, the fuzzy congestion controller produce a traffic jam indicator $\mathrm{y}$ and the system information statistics measure the cell loss ratio $\mathrm{P}_{\mathrm{t}}$ which handles the CAC function. 
The acceptance and rejection of new calls request based on decision signal Z, which is sent by NFCAC controller, define as following

If $\mathrm{P}_{\mathrm{t}}<\mathrm{QoS}$

Then

Accept the new call request and set $\mathrm{Z}=1$.

Else

Reject the new call request and set $\mathrm{Z}=0$.

Now for the confirmation of the acceptance decision of a new call, we carry on the simulation process for a fixed time interval, without receiving any new call request in the network. From the simulation result, we acquire numerical data of cell loss ratio $P_{t}^{\prime}$ If $P_{t}^{\prime}>$ QoS, the new call is rejected, then Set $\mathrm{Z}=0$.

This training data is stored in $B_{a}, y, \mathrm{Pt}$, and $\mathrm{Z}$ for further use as inputs $\left(B_{a}, y, \mathrm{Pt}\right)$ and desired output $(\mathrm{Z})$. The fuzzy membership function and the fuzzy control rules are obtained by the fuzzy partitioned parameters and the self - organized training. If the first training set is applied to design the initial set of fuzzy control rules, then a number of feasible control rule set can be created by minimal changes in fuzzy rules. From all the possible set of rules, our model select one of control rule set for training data that gives the minimum square error $\mathrm{E}$ defined as follow

$$
E=\frac{1}{2} \sum_{i=1}^{N}\left[z\left(t_{i}\right)-z^{-}\left(t_{i}\right)\right]^{2}
$$

The above equation state that after applying the $\mathrm{N}$ number of tanning data set at the time $t_{i}$ the desired output $z\left(t_{i}\right)$ and the actual output $z^{-}\left(t_{i}\right)$ are obtained.

Kohonen's SOM feature-maps and N-nearest neighbor's methods are used to approximate the initial position of the membership functions if the training set is not provided initially [1]. If the initial position of the NFCAC membership functions is estimated then means $m_{i}$ value of the every $i^{\text {th }}$ the membership function is calculated for a given set of linguistic input tanning data $x_{i}$ for $x$ as described in the given algorithm. Here statistical grouping method of Kohonen's SOM feature-maps algorithm is used, described as below.

[Obtain mean $\left(m_{i}\right)$ value by using SOM featured map algorithm]

Step 1: Initialize the random values of $m_{i}$ for all NFCAC membership function $(1 \leq i \leq M)$ and the value of $m_{i}$ should be lies between as given below

$$
\min _{1 \leq i \leq N} x_{i} \leq m_{i} \leq \max _{1 \leq i \leq N} x_{i} \text {, set } x=1
$$

Step 2: Set the initial learning rate $\alpha(0<\alpha<1)$, set $k=1$.

Step 3: Calculate the actual distance $d_{i}$ (Winning Neurons) by applying the tanning data set $x_{i}$

$$
d_{i}=\left|x_{i}-m_{i}\right|, 1 \leq i \leq M .
$$

Step 4: In this step, decide $k^{t h}$ membership function from the available set membership function, which contain minimum distance $d_{k}$ (i.e. $d_{k}=\min 1 \leq i \leq M d_{i}$ ) Now update $M_{k}$ by

$$
M_{k}=M_{k}+\alpha\left(x_{j}-m_{k}\right) \text {. }
$$

Step 5: Check if $k<N$, then set $k=k+1$ and GOTO

Step 3 Otherwise, Decrease $\alpha$ and GOTO Step 2.

Step 6: Repeat step from 1 to 5 until $\alpha \leq 0$.

In step 4 , a winner-take-all circuit determines which one $d_{i}$ is minimum to get $m_{i}$ for all input/ output parameters [33].
Now after the successful completion of the SOM training phase, the NFCAC controller goes into the supervised learning phase. For supervise learning error back-propagation (EBP) method of NN is used to reduce the error $E$ for training data set. Further, from the output node, a feedback path is applied to calculate $\partial E / \partial w$ for all hidden layer nodes used in layer 4 and layer 2 . The common learning rule applied for the weight adjustment $w$ in the hidden nodes are as follow

$$
\mathrm{w}^{\text {new }}=\mathrm{w}^{\text {old }}+\eta \frac{\partial \mathrm{E}}{\partial \mathrm{w}}
$$

Here $\eta$ is the learning rate and the variation of $\eta$ values are employed in Layer 2 and layer 4 to deliver quality learning rate for input and output variables set. The value of $\eta$ will be zero when there is no need to update the membership function for a particular input variables.

\section{SIMULATION RESULTS AND DISCUSSION}

A very high-speed wireless multimedia network (like LTE/4G) is chosen for the simulation of NFCAC model. In this input traffic is defined into two classes: class-1-real-time (Voice), provides the services for both new and handoff voice call while class-2-nonreal-time (Data), provides the services of data calls. Based on the prior information about CAC schemes, initially, the rule formation and input variables of the NFCAC controller are set and modified via the NN learning algorithm. The fuzzy membership function of a given set of input variables for class- 1 and class- 2 traffic are shown in Fig. 5(a) and Fig. 5(b), respectively. According to equation (1), the bandwidth used by the new calls and existing calls is subtracted from the total available bandwidth. So as to keep maintain the service quality for the user anywhere and anytime, the NFCAC controller manages the bandwidth according to traffic types of the networks. If a network system has a total bandwidth $B_{t}$, then $B_{u}$ bandwidth is reserved for class- 1 and the remaining $\left(B_{t}-B_{u}\right)$ bandwidth are reserved for class- 2 . In order to the proper utilization of network (bandwidth), our system use the channel borrowing scheme to assign more bandwidth to the required class of traffic. So that the mean value of the initial membership function of the available bandwidth $B_{a}$ is set (i.e. $m_{11}^{(I)}$ is set for Low (L), $m_{12}^{(I)}$ is set for Medium (M) and $m_{13}^{(I)}$ is set for High $(\mathrm{H})$. Further, during the simulation of the NFCAC model the performance of the network traffic jam indicator $y$ could be examined by the three states of the wireless network, congestion full (H), partially congestion (M) and congestion free (L). Thus the obtained information can be used to optimized the initial membership function of $y$. The mean value $m_{j n}^{(I)}$ is set for the congestion full $(\mathrm{H})$, partially congestion (M) and congestion free (L) states of the wireless networks (i.e. $m_{21}^{(I)}$ for $(\mathrm{H}), m_{22}^{(I)}$ for $(\mathrm{M})$ and $m_{23}^{(I)}$ for $(\mathrm{L})$ ). On the bases of predefined QoS parameters the primary 
membership function of the cell loss ratio $P_{t}$ is set and obtain the mean value of the membership function, (i.e. the mean value $m_{31}^{(I)}$ is set when the QoS requirements are not satisfied $(\mathrm{H}), m_{32}^{(I)}$ is set when the QoS requirements are partially satisfied $(\mathrm{M})$, and $m_{33}^{(I)}$ is set when the QoS requirements are satisfied $(\mathrm{L})$, the user requirements). Now based on the fuzzy membership function the initial value of the output term set $T\left(z^{-}\right)$for the mean value $m_{j}^{(O)}$ is set. The value of $m_{j}^{(0)}$ is set between the range of 0 and 1 . When the first phase of simulation is completed an optimal set of fuzzy rule structure are obtained (as shown in Table 1), then the NFCAC model goes into supervise learning phase. Here all the obtained membership functions are modified optimally in order to maintain the relationship between the previous and modified membership functions.

The obtained membership function of the input linguistic variables for class-1 (voice) and class-2 (Data) traffic is represented in fig. 4 (a) and 4 (b). In supervise learning phase there is a need the optimization of the initial fuzzy membership function of $B_{a}$ and $z^{-}$, as were heuristically set. For class-1 type traffic the fig. 4(a) shown the membership functions, which is defined as follow. For the membership function of $B_{a}$, the mean value $m_{11}^{(I)}, m_{12}^{(I)}$ and $m_{13}^{(I)}$ of the membership function, $\mathrm{L}, \mathrm{M}$ and $\mathrm{H}$ are assigned. As we obtain the membership function of $y$, then a drastic change have been made in the membership function of $B_{a}$, which reduce the training time in supervise learning phase. Here a set of three values of $\eta\left\{p_{t}=0, \mathrm{y}=0.001, B_{a}, z^{-}=0.01\right\}$ is define for the variables $p_{t}, \mathrm{y}, B_{a}$ and $z^{-}$respectively. The membership function of $p_{t}$ will be remain unchanged because the value of $\eta$ is zero for $p_{t}$. Now we will examine the membership functions of the output variable $Z^{-}$, the mean values $m_{1}^{(O)}$ and $m_{3}^{(O)}$ of the membership function $R$ and $W A$ are slightly increases, from 0 to 0.05 and from 0.60 to 0.67 , respectively. The mean values of $R$ and $W A$ shows that the effects of the rejection and weakly acceptance are slightly increased. The output variable $z^{-}$is the set of four terms, if we apply some changes in the location of any one terms of $z^{-}$, then it will take more attentions from others three unchanged variables. This change will compressed the functionality of the remaining three variables, so the change of the location can only be made in a small range. After this change in the membership function of $z^{-}$, the NFCAC controller is more capable to accept new and handoff calls by recovering some unused bandwidth in the system in order to maintain the QoS parameters. The similar phenomenon can be applied to obtain the results for class-2 traffic in Fig. 4(b).

Table 1: FIS generated IF-THEN fuzzy rules

\begin{tabular}{|c|c|c|c|c|}
\hline \multicolumn{5}{|c|}{ Explanation of IF-THEN rules } \\
\hline & IF & & & THEN \\
\hline $\begin{array}{l}\text { Rule } \\
\text { No. }\end{array}$ & $B_{a}$ & $y$ & $P_{t}$ & $z^{-}$ \\
\hline 1 & $\mathrm{~L}$ & $\mathrm{H}$ & $\mathrm{H}$ & $\mathrm{R}$ \\
\hline 2 & $\mathrm{~L}$ & $\mathrm{H}$ & $M$ & $\mathrm{R}$ \\
\hline 3 & $L$ & $\mathrm{H}$ & $\mathrm{L}$ & WR \\
\hline 4 & $\mathrm{~L}$ & $M$ & $\mathrm{H}$ & $R$ \\
\hline 5 & $\mathrm{~L}$ & $M$ & $M$ & $R$ \\
\hline 6 & $\mathrm{~L}$ & $\mathrm{M}$ & $\mathrm{L}$ & WA \\
\hline 7 & $\mathrm{~L}$ & $\mathrm{~L}$ & $\mathrm{H}$ & WA \\
\hline
\end{tabular}

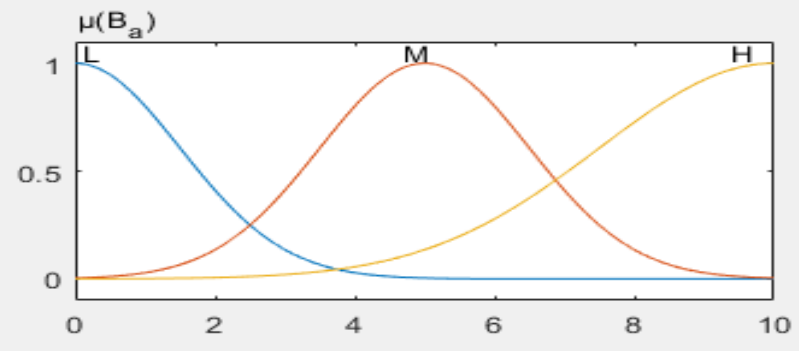

$\mu(\mathrm{y})$
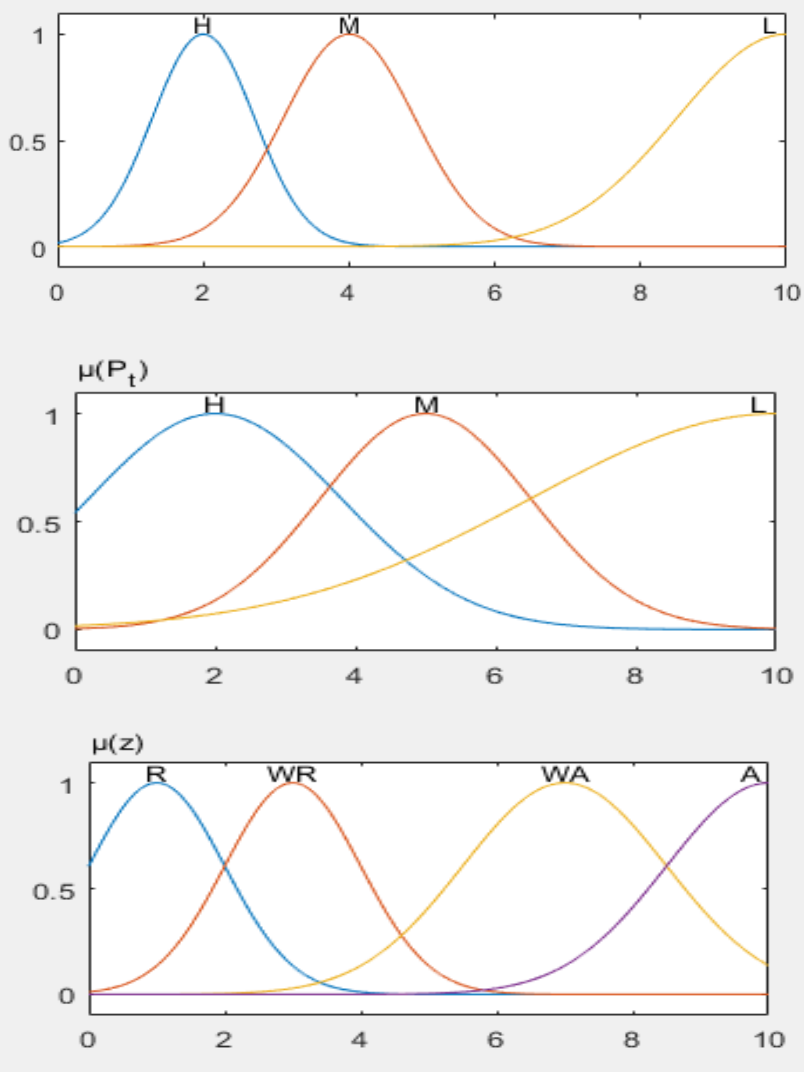

(a)

Published By:

Blue Eyes Intelligence Engineering \& Sciences Publication 

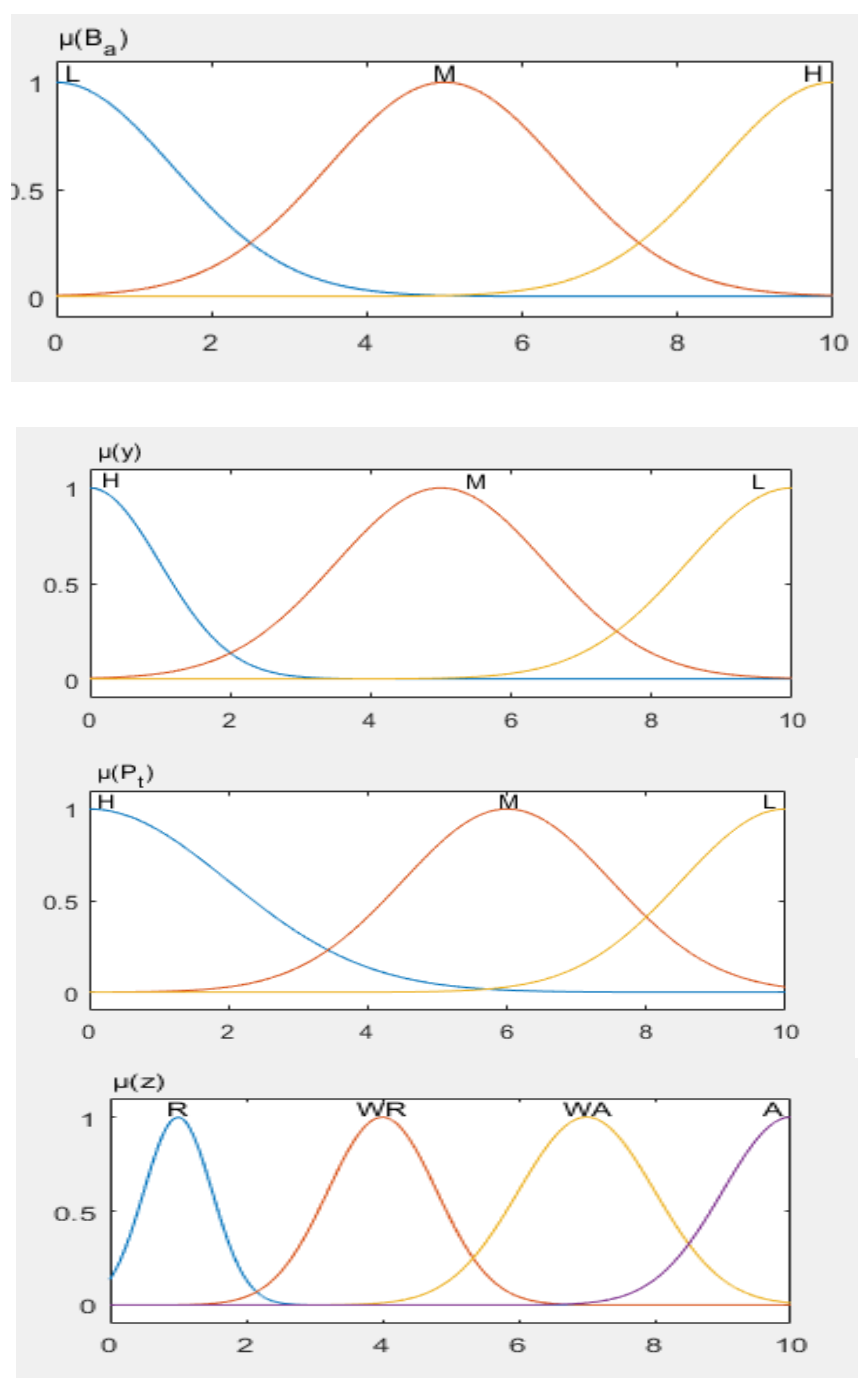

(b)

Fig.4 Membership function of $B_{a}, y, P_{t}$ and $z$ for (a) Class-1(Voice) and (b) Class-2 (Data)

The simulation results of NFCAC model is compared with efficient resource allocation based CAC (ERCAC) schemes as given in [39], the FL based CAC (FLCAC) policy given in [18-19], the neural network based CAC (NNCAC) as developed in [25-26] and the radial basis function-based CAC (RBFCAC) scheme in terms of cell loss ratio (CLR), channel utilization, call dropping probability (CDP) and call blocking probability (CBP) under the constraint of QoS guarantee.

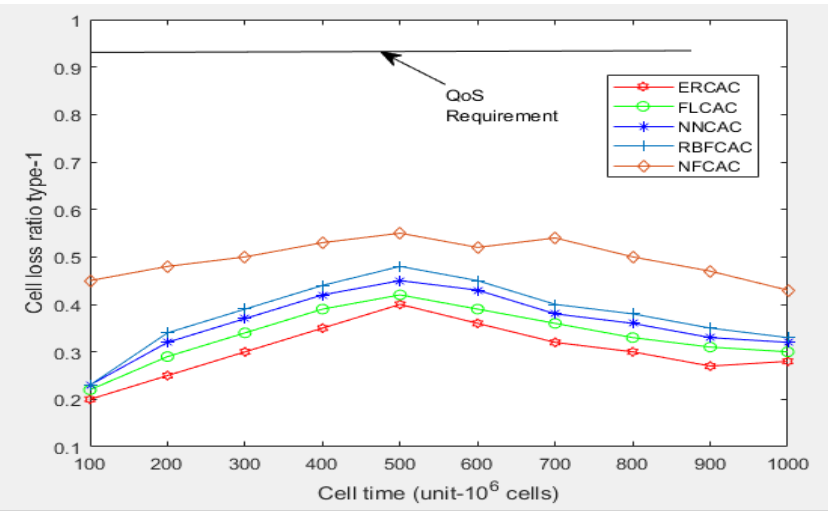

Fig. 5. (a). Cell loss ratio for class-1 (Voice) traffic

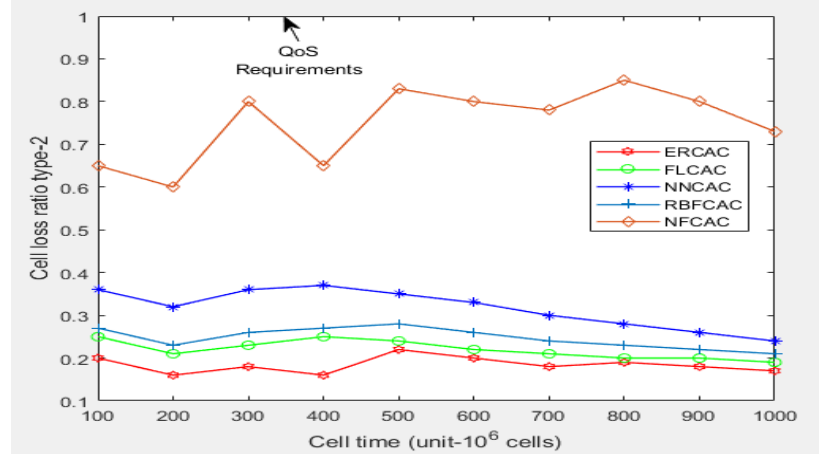

Fig. 5 (b). Cell loss ratio for class-2 (Data) traffic.

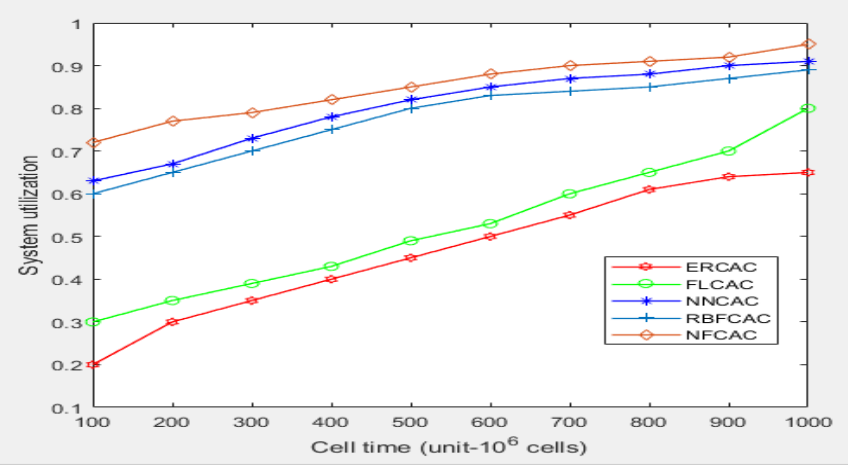

Fig. 6. System resource utilization.

The cell loss ratios (CLR) of a mobile traffic controller implemented in the proposed (NFCAC) model and other call admission control schemes like ERCAC, FLCAC, NNCAC, RBFCAC are shown in Fig. 5. It has been seen that it maintain the guaranteed QoS level for both types of traffic services. Fig. 6 shows the utilization of the system resource by the NFCAC and the other four CAC policies. It is observed that the percentage of system utilization of the proposed NFCAC is greater than the NNCAC, RBFCAC and much higher than FLCAC and ERCAC. The system utilization percentage of NFCAC, NNCAC and RBFCAC are 94\%, 91\%, and 89\% respectively, and the NFCAC provide $36 \%$ to $15 \%$ higher system utilization than the previously existing schemes EBCAC and FLCAC. This is because of NFCAC model has self-training ability of the neural network and rule structured ability of fuzzy logic.

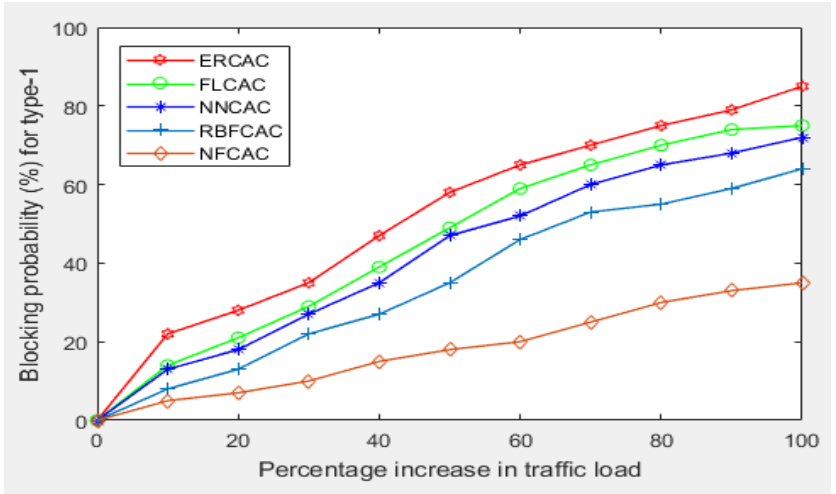

(a) 


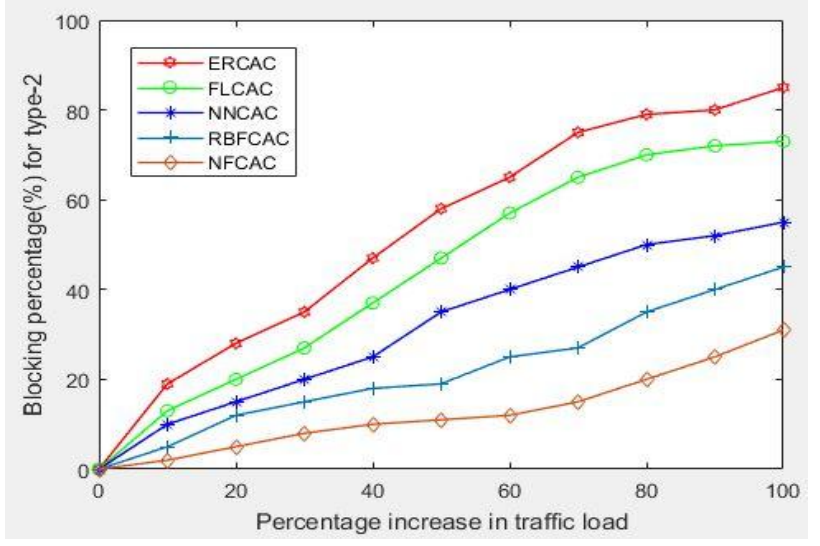

(b)

Fig. 7. Call blocking (\%) for (a) class-1 (Voice) traffic and (b) class-2 (Data) traffic.

Fig. 7 (a) and (b), shows the performance of NFCAC in terms of call blocking probabilities under different traffic load for both types of mobile network services (real-time and non-real time). According to the simulation result, the blocking probabilities in both service class are lesser than the compared CAC policies. NFCAC scheme can accept a new call only when the admission criteria match with FIS rules.

According to Fig. 8 (a) and (b), the dropping probability obtained in both types of mobile traffic by NFCAC model is lesser than NNCAC, RBFCAC, FLCAC and ERCAC schemes. After the simulation, it has been observed that the dropping probability percentage of NFCAC is lesser than about to $20 \%$, 33\%, and 39\% from RBFCAC, NNCAC and FLCAC respectively.

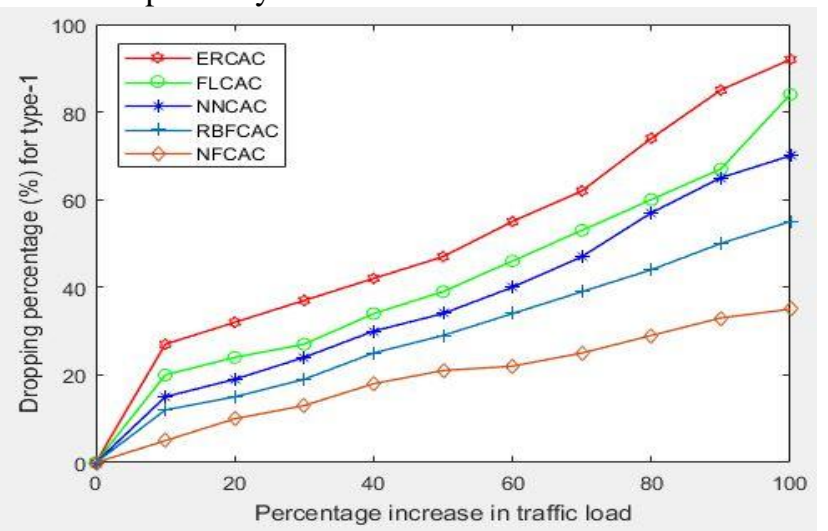

(a)

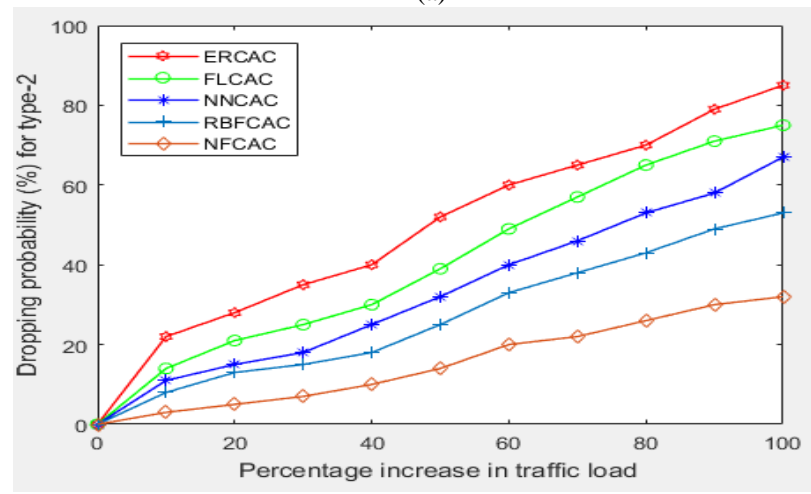

(b)

Fig. 8. Call dropping (\%) for (a) class-1 (voice) traffic and (b) class-2 (data) traffic

\section{CONCLUSION}

This paper proposed a neuro-fuzzy call admission control (NFCAC) model for high-speed mobile multimedia networks. The main objective of this integrated neural fuzzy based CAC scheme is to maintain quality of service with proper resource allocation, in order to minimize the CDP and CBP in mobile multimedia networks. The proposed neural fuzzy CAC scheme is a hybrid approach that merges the semantic control abilities of FL controller and self-training capabilities of a NN to construct an efficient computational model for traffic control and fair channel allocation for new calls and handoff calls. The proposed NFCAC model, implemented in the two-phase learning method. The self-organized learning scheme is used in the first phase for the construction of fuzzy rules and membership functions, while the second phase adopts supervised learning scheme for the ideal adjustment of the membership functions to attain desired outputs in terms of acceptance and rejection of calls, which maintains the QoS requirements at different traffic load. According to the simulation results, the system utilization percentage of NFCAC, NNCAC and RBFCAC are $94 \%, 91 \%$, and $89 \%$ respectively, and the NFCAC provide $36 \%$ to $15 \%$ higher system resource utilization than the previously existing schemes EBCAC and FLCAC. The dropping probability percentage of NFCAC is lesser than about to $20 \%$, 33\%, and 39\% from RBFCAC, NNCAC and FLCAC respectively.

\section{REFERENCES}

1. Ray-Guang Cheng, Chung-Ju Chang and Li-Fong Lin," A QoS provisioning neural fuzzy connection admission controller for multimedia high-speed networks," in IEEE/ACM Transactions on Networking, vol. no. 1, 1999, pp.111-121.

2. Roberto Sepúlveda, Oscar Montiel-Ross, Jorge Quiñones-Rivera, and Ernesto E. Quiroz, "WLAN Cell Handoff Latency Abatement Using an FPGA Fuzzy Logic Algorithm Implementation," Advances in Fuzzy Systems, vol. 2012.

3. S. Kumar, K. Kumar and K. Pandey, "A Comparative Study of Call Admission Control in Mobile Multimedia Networks using Soft Computing". International Journal of Computer Applications (0975 8887) Vol. 107 Issue 16, 2014, pp. 1-7.

4. P. B. Metre, K. R. Radhika and Gowrishankar, "Survey of soft computing techniques for joint radio resource management," 2012 International Conference on Multimedia Computing and Systems, Tangier, 2012, pp. 562-565.

5. P. Payaswini, D.H. Manjaiah "Challenges and issues in $4 \mathrm{G}-$ Networks Mobility Management”.International Journal of Computer Trends and Technology (IJCTT) - volume4 Issue5, 2013, pp.1247-1251.

6. A. A. Atayero and M. K. Luka 'Applications of Soft Computing in Mobile and Wireless Communications". International Journal of Computer Applications (0975 - 8887), vol. 45 No. 22, 2012, pp. 48-55,

7. I. F. Akyildiz, D. M. Gutierrez-Estevez, E. C. Reyes "The evolution to 4G cellular systems". LTE Advanced Physical Communication, vol. 3 , 2010, pp. 217-244.

8. A. Shukla, Super-Fast 4G Wireless Service Launching in South Korea. Asia-Pacific Business and Technology Report, 2011.

9. G. Mahesh, S. Yeshwanth, U. V. Manikantan "Survey on Soft Computing based Call Admission Control in Wireless Networks". International Journal of Computer Science and Information technology, Vol. 5 No. 3, 2014, pp. 3176 - 3180.

10. Technologies, Vol. 5 No. 3, pp 3176 - 3180, 2014. W. Wang, X. Wang, and A. A. Nilsson, "Energy- Efficient Bandwidth Allocation inWireless Networks: Algorithms, Analysis, and Simulations", IEEE Transactions onWireless Communications, Vol 5, No. 5, 2006, pp. 1103-1114.

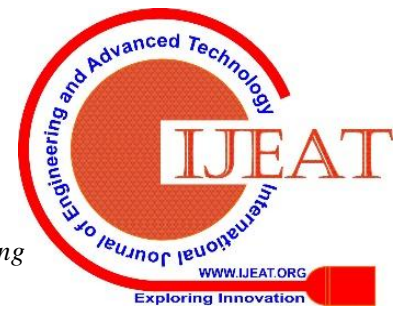


11. O.Castillo and P.melin,'Type-2fuzzy logic: theory and application", stud fuzz, vol.233, 2008, pp.29-43.

12. A. R. Bonde and S. Ghosh, "A comparative study of fuzzy versus 'fixed' thresholds for robust queue management in cell-switching networks," IEEE/ACM Trans. Networking, vol. 2, 1994, pp. 337-344.

13. Ravi sindal and sanjiv Tokekar "A Neuro-Fuzzy Call Admission Control Algorithm for voice /data traffic in CDMA Cellular Network" IEEE Interantional Advance Computing Conference (IACC-2009), Patiala, India, march 2009.

14. Raad Raad and Ibrahim Raad,'Neuro-Fuzzy Admission Control in Cellular Networks" 10th IEEE International Conference on Communication Systems (ICCS 2006), Singapore, November 2006.

15. Ramesh Babu H.S, Gownshankar and Satyanarayana P.S," An Intelligent Fuzzy Neural Call Admission Control Mechanism for Guaranteed QoS in Heterogeneous Wireless Networks”, Proceedings of the International Multi-Conference of Engineers and Computer Scientists (IMECS-2010), Vol. 1, Hong Cong, 2010.

16. C. T. Lin and C. S. G. Lee, "Neural fuzzy systems", Prentice Hall 1996.

17. Ramesh Babu H.S., Gowrishankar, Satyanarayana P," A QoS Provisioning Recurrent Neural Network based Call Admission Control for beyond 3G Networks", IJCSI International Journal of Computer Science Issues, ISSN-1694-0784, Vol. 7, Issue 2, No 5,2010.

18. Gjergji Mino, Leonard Barolli, Arjan Durresi, Fatos Xhafa, and Akio Koyama," Fuzzy-based Call Admission Control Scheme for Wireless Cellular Networks Considering Priority of On-going Connections" ,29th IEEE International Conference on Distributed Computing Systems Workshops, Montreal, Canada ,June 2009.

19. Sakamoto, and Leonard Barolli," A Fuzzy-based CAC Scheme for Cellular Networks Considering Security”, International Conference on Network-Based Information Systems, IEEE 2014.

20. Uduak Umoh, Daniel Asuquo, and Imoh Eyoh," An Interval type-2 Fuzzy Logic based Framework for Call Admission Control in 4G Mobile Network", 2017, pp. 23-36.

21. M. V. Ramkumar, A. D. Mihovska, N. R. Prasad and R. Prasad "Fuzzy-Logic Based Call Admission Control for A Heterogeneous Radio Environment". International Jornal of Research in Computer Science, vol. 3, Issue 4, 2016.

22. M. P. Selvi and S. Sendhilnathan "Fuzzy-Based Mobility Management in 4G Wireless Networks". Brazilian Archives of Biology and Technology. vol. 59, 2016.

23. G. U. Mali "Fuzzy-Based Vertical Handoff Decision Controller for Future Networks". International Journal of Advanced Engineering, Management and Science (IJAEMS) vol. 3, no.1, 2017, pp.111-119.

24. J. Ye, X. Shen, and J. Mark, "Call admission control in wideband CDMA cellular networks by using fuzzy logic". IEEE Trans. Mobile Comput. vol. 4, no. 2, 2005, pp. 129-141.

25. S. Kumar, K. Kumar and P. Kumar, "Mobility based call admission control and resource estimation in mobile multimedia networks using artificial neural networks," 2015 1st International Conference on Next Generation Computing Technologies (NGCT), Dehradun, 2015, pp. 852-857.

26. Kumar Sanjeev, Krishan Kumar, and Anand Kumar Pandey, "Dynamic channel allocation in mobile multimedia networks using Error Back Propagation and Hopfield Neural Network (EBP-HOP)", Procardia Computer Science, vol. 89:, 2016, pp.107-116.

27. Sanjeev Kumara, Krishan Kumara, and Anand Kumar Pandey," Hopfield Neural Network based Dynamic Call Admission Control for Quality of Service Provisioning in Mobile Multimedia Networks" International Journal of Information Processing, 10(3), 2016, pp.88-97.

28. D. Liu, Y. Zhang, H. Zhang, "A self-learning call admission control scheme for CDMA cellular networks," In IEEE Transactions on Neural Networks, vol.16, no. 5, ,2005, pp. 1219-1228.

29. J. Qi, F. Wu, L. Li, H. Shu, "Artificial intelligence applications in the telecommunication industry," in Expert Systems, vol. 24, no. 4, 2007, pp. 271-291.

30. Lotfi A. Zadeh. Fuzzy Logic, Neural Networks, and Soft Computing", Communications of the ACM, Vol. 37.

31. B. Kosko, Neural Networks, and Fuzzy Systems, Prentice-Hall, 1992.

32. Shih-Cheng Horng1 and Shieh-Shing Lin," Fuzzy call admission control combined with distributed dynamic channel assignment and reassignment for cellular mobile systems", EURASIP Journal on Wireless Communications and Networking 2015.

33. C. T. Lin and C. S. G. Lee, "Neural-network-based fuzzy logic control and decision system," IEEE Trans. Comput., vol. 40, 1991, pp. 1320-1336.

34. Smitha. K1, Anand H. U.2, and J. D. Mallapur," Fuzzy Logic Based Congestion Control in Wireless Networks", International Journal of Computer Science and Communication Vol. 2, No. 2, 2011, pp. 469-473.
35. Chrysostom Ou C. and Pitsillides A. "Fuzzy Logic Control in Communication Networks. In: Hassanien AE., Abraham A., Herrera F. (eds) Foundations of Computational Intelligence Volume 2. Studies in Computational Intelligence, vol 202. Springer, Berlin, Heidelberg, 2009.

36. Chenn-Jung Huang and Wei-Kuang Lai, "Application of the neuro-fuzzy technique to the bandwidth reservation for sectored cellular communication," IEEE Trans.,2003, pp 958- 965.

37. L. T. W. Ho, G. S. Louis, and M. P. Jonathan, "Applying emergent self-organizing behavior for the coordination of $4 \mathrm{G}$ networks using complexity metrics," Bell Labs Tech. J., vol. 8, no. 1, 2003, pp. 5-25.

38. M. Duque-Antón, B. Rüber, U. Killat, "Extending Kohonen's Self-Organizing Mapping for Adaptive Resource Management in Cellular Radio Networks", IEEE Transactions on Vehicular Technology, vol. 46, no. 3,1997, pp. 560-568

39. Chi Wa Leong, Weihua Zhuang, Yu Cheng, and Lei Wang, "Optimal Resource Allocation and Adaptive Call Admission Control for Voice/Data Integrated Cellular Networks", IEEE transactions on vehicular technology, vol. 55, no. 2,2006.

\section{AUTHORS PROFILE}

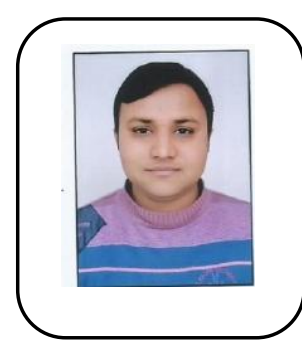

Sanjeev Kumar, received the Master of Computer Application Degree from Utter Pradesh Technical University, Lucknow in 2007.He is research scholar in the Department of Computer Science at Gurukul Kangri Vishwavidyalaya, Hardwar. He has qualified UGC-NET in Computer Science and Application. He has published 12 research papers in several International/National Conference and Journals. His research interests are in Wireless Communication, Artificial Neural Networks and its applications.

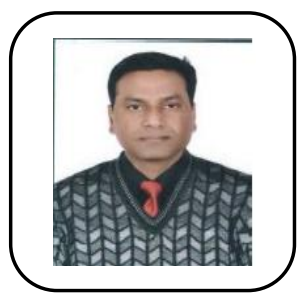

Krishan Kumar, is presently working as Assistant Professor in the Department of Computer Science, Faculty of Technology, Gurukula Kangri Vishwavidyalaya, Haridwar, India. He obtained his MCA and Ph.D in Computer Science and Information Technology from Institute of Engineering and Technology, MJP Rohilkhand University, Bareilly. He has qualified UGC-NET in Computer Science and Application. He has published 30 research papers in several International/National Conference/Journals. $\mathrm{He}$ is an active member of various societies and also the Chairman of Computer Society of India, Haridwar Chapter. His principal research area is Artificial Neural Networks and its applications. 